\title{
Research Article \\ Dynamics of Cohen-Grossberg Neural Networks with Mixed Delays and Impulses
}

\author{
Xinsong Yang, ${ }^{1,2}$ Chuangxia Huang, ${ }^{1,3}$ Defei Zhang, ${ }^{2}$ and Yao Long ${ }^{2}$ \\ ${ }^{1}$ Department of Mathematics, Southeast University, Nanjing 210096, China \\ ${ }^{2}$ Department of Mathematics, Honghe University, Mengzi, Yunnan 661100, China \\ ${ }^{3}$ Department of Mathematics, College of Mathematics and Computing Science, \\ Changsha University of Science and Technology, Changsha, Hunan 410076, China
}

Correspondence should be addressed to Chuangxia Huang, cxiahuang@126.com

Received 8 September 2008; Accepted 5 November 2008

Recommended by Ülle Kotta

Impulsive Cohen-Grossberg neural networks with bounded and unbounded delays (i.e., mixed delays) are investigated. By using the Leray-Schauder fixed point theorem, differential inequality techniques, and constructing suitable Lyapunov functional, several new sufficient conditions on the existence and global exponential stability of periodic solution for the system are obtained, which improves some of the known results. An example and its numerical simulations are employed to illustrate our feasible results.

Copyright (C) 2008 Xinsong Yang et al. This is an open access article distributed under the Creative Commons Attribution License, which permits unrestricted use, distribution, and reproduction in any medium, provided the original work is properly cited.

\section{Introduction}

In the recent years, dynamics of the Cohen-Grossberg neural networks (CGNNs) [1] has been extensively studied because of their immense potentials of application perspective in different areas such as pattern recognition, parallel computing, associative memory, combinational optimization, and signal and image processing [2-6]. The authors of [7-11] have studied the stability of equilibrium point or periodic solution of CGNNs with timevarying delays due to the transmission delays during the communication between neurons which will affect the dynamical behavior of neural networks. Considering the distant past also has influence on the recent behavior of the state, the authors of [12] investigated the stability of equilibrium point for CGNNs with continuously distributed delays. Impulsive effects are also likely to exist in the neural networks, that is, the state of the networks is subject to instantaneous perturbations and experiences abrupt change at certain moments. Authors of [13-16] have studied the stability of equilibrium point for impulsive delay CGNNs. However, the activation functions of CGNNs in [12-16] are bounded, and the restrictions on impulses in $[13,17]$ are very strong, which limit CGNNs' applications [7]. 
In theory and applications, global stability of periodic solution of CGNNs is of great importance since the global stability of equilibrium points can be considered as a special case of periodic solution with random period. Moreover, CGNNs model is one of the most popular and typical neural network models. Some other models, such as Hopfield-type neural networks, cellular neural networks, and bidirectional associative memory neural networks, are special cases of the model [14]. To our best knowledge, few authors have considered the existence and global exponential stability of periodic solutions for CGNNs with mixed delays and impulses. Therefore, it is necessary to consider the existence and global exponential stability of periodic solution for impulsive CGNNs with mixed delays.

The main methods used in this paper are Leray-Schauder's fixed point theorem, differential inequality techniques, and Lyapunov functional. Several new sufficient conditions are obtained for the existence and global exponential stability of periodic solution for impulsive CGNNs with mixed delays. Moreover, we discharge some restrictive conditions on the activation functions of the neurons, such as boundedness, monotonicity, and differentiable, we offer the precise convergence index, and give the weak conditions on impulses.

The rest of this paper is organized as follows. In Section 2, we introduce some notations and definitions and state some preliminary results needed in later sections. We then study, in Section 3, the existence of periodic solutions of impulsive CGNNs with mixed delays by using Leray-Schauder's fixed point theorem. In Section 4, with the help of Lyapunov functional, we will derive sufficient conditions for the global exponential stability of the periodic solution. At last, an example and its numerical simulations are employed to illustrate the feasible results of this paper.

\section{Preliminaries}

Consider the following CGNNs with mixed delays and impulses:

$$
\begin{aligned}
\begin{aligned}
u_{i}^{\prime}(t)=-a_{i}\left(u_{i}(t)\right)\left\{b_{i}\left(u_{i}(t)\right)-\sum_{j=1}^{n}[\right. & b_{i j}(t) f_{j}\left(u_{j}\left(t-\tau_{i j}(t)\right)\right)
\end{aligned} \\
\left.\left.\quad+c_{i j}(t) \int_{-\infty}^{t} k_{i j}(t-s) g_{j}\left(u_{j}(s)\right) \mathrm{d} s\right]+I_{i}(t)\right\}, \quad t \neq t_{k}, \\
\Delta u_{i}\left(t_{k}\right)=u_{i}\left(t_{k}^{+}\right)-u_{i}\left(t_{k}^{-}\right)=\gamma_{i k} u_{i}\left(t_{k}\right), \quad i=1,2, \ldots, n,
\end{aligned}
$$

where $\Delta u_{i}\left(t_{k}\right)(i=1,2, \ldots, n)$ are the impulses at moments $t_{k}, u_{i}(t)$ is left continuous at time $t_{k}$, and the right limit exists at $t_{k}$, that is, $u_{i}\left(t_{k}\right)=u_{i}\left(t_{k}^{-}\right)$and $u_{i}\left(t_{k}^{+}\right)$exist and $0<t_{1}<t_{2}<\cdots$ is a strictly increasing sequence such that $\lim _{k \rightarrow+\infty} t_{k}=+\infty ; u_{i}(t)$ is the state of neuron; $a_{i}\left(u_{i}(t)\right)$ and $b_{i}\left(u_{i}(t)\right)$ represent an amplification function at time $t$ and an appropriately behaved function at time $t$, respectively; $B=\left(b_{i j}(t)\right)_{n \times n}$ and $C=\left(c_{i j}(t)\right)_{n \times n}$ are connection matrices; $I(t)=\left(I_{1}(t), I_{2}(t), \ldots, I_{n}(t)\right)^{T}$ is the input function; and $g(u)=\left(g_{1}\left(u_{1}\right), g_{2}\left(u_{2}\right), \ldots, g_{n}\left(u_{n}\right)\right)^{T}$ are the activation functions of the neurons.

Throughout this paper, we assume that

$\left(\mathrm{H}_{1}\right) b_{i j}(t), c_{i j}(t), \tau_{i j}(t) \geq 0, I_{i}(t)$ are continuous $\omega$-periodic functions, $\omega>0$ is a constant, $i, j=1,2, \ldots, n$

$\left(\mathrm{H}_{2}\right) a_{i}(u)$ is continuous and there exist positive constants $\underline{a}_{i}$ and $\bar{a}_{i}$ such that $0<\underline{a}_{i} \leq$ $a_{i}(u) \leq \bar{a}_{i}, u \in R, i=1,2, \ldots, n$; 
$\left(\mathrm{H}_{3}\right)$ there is a positive constant $l_{i}$ such that $b_{i}^{\prime}(u) \geq l_{i}, b_{i}^{\prime}(u)$ denotes the derivative of $b_{i}(u), u \in R$, and $b_{i}(0)=0, i=1,2, \ldots, n$;

$\left(\mathrm{H}_{4}\right)\left\{\gamma_{i k}\right\}$ and $\left\{t_{k}\right\}$ are $\omega$-periodic sequence, that is, there exists a positive constant $p$ such that $t_{(k+p)}=t_{k}+\omega, \gamma_{i(k+p)}=\gamma_{i k}, i=1,2, \ldots, n$;

$\left(\mathrm{H}_{5}\right) f_{j}, g_{j} \in C(R, R)$, there are positive constants $L_{j}^{f}>0, L_{j}^{g}>0$, such that $\left|g_{j}(x)-g_{j}(y)\right| \leq$ $L_{j}^{g}|x-y|,\left|f_{j}(x)-f_{j}(y)\right| \leq L_{j}^{f}|x-y| \forall x, y \in R, j=1,2, \ldots, n ;$

$\left(\mathrm{H}_{6}\right)$ the delay kernels $k_{i j}:[0,+\infty) \rightarrow R$ are continuous, integrable and there exist positive constants $\beta_{i j}$ such that

$$
\int_{0}^{+\infty}\left|k_{i j}(s)\right| \mathrm{d} s \leq \beta_{i j}, \quad i, j=1,2, \ldots, n ;
$$

$\left(\mathrm{H}_{7}\right)$

$$
\begin{gathered}
\max _{1 \leq i \leq n} \sup _{0 \leq t \leq \omega}\left\{\frac{\sum_{j=1}^{n} \bar{a}_{j}\left[\left|b_{i j}(t)\right| L_{j}^{f}+\left|c_{i j}(t)\right| L_{j}^{g} \beta_{i j}\right]}{q_{i}}\right\}=\theta<1, \\
\max _{1 \leq i \leq n}\left\{\theta+\frac{\bar{a}_{i}}{\underline{a}_{i}\left(1-e^{-q_{i} \omega}\right)} \sum_{k=1}^{p}\left|b_{i k}\right|\right\}<1,
\end{gathered}
$$

where $q_{i}=\underline{a}_{i} l_{i} ;$

$\left(\mathrm{H}_{8}\right)$ there exists a constant $\alpha_{0}>0$ such that

$$
\int_{0}^{+\infty}\left|k_{i j}(s)\right| e^{\alpha_{0} s} \mathrm{~d} s<+\infty, \quad i, j=1,2, \ldots, n
$$

From $\left(\mathrm{H}_{2}\right)$, the antiderivative of $1 / a_{i}\left(u_{i}\right)$ exists. We choose an antiderivative $h_{i}\left(u_{i}\right)$ of $1 / a_{i}\left(u_{i}\right)$ that satisfies $h_{i}(0)=0$. Obviously, $\left(\mathrm{d} / \mathrm{d} u_{i}\right) h_{i}\left(u_{i}\right)=1 / a_{i}\left(u_{i}\right)$. By $a_{i}\left(u_{i}\right)>0$, we obtain that $h_{i}\left(u_{i}\right)$ is strictly monotone increasing about $u_{i}$. In view of derivative theorem for inverse function, the inverse function $h_{i}^{-1}\left(u_{i}\right)$ of $h_{i}\left(u_{i}\right)$ is differential and $\left(\mathrm{d} / \mathrm{d} u_{i}\right) h_{i}^{-1}\left(u_{i}\right)=a_{i}\left(u_{i}\right)$. By $\left(\mathrm{H}_{3}\right)$, composition function $b_{i}\left(h_{i}^{-1}(z)\right)$ is differential. Denote $x_{i}(t)=h_{i}\left(u_{i}(t)\right)$, it is easy to see that $x_{i}^{\prime}(t)=u_{i}^{\prime}(t) / a_{i}\left(u_{i}(t)\right)$ and $u_{i}(t)=h_{i}^{-1}\left(x_{i}(t)\right)$. Substituting these equalities into system (2.1), we get

$$
\begin{aligned}
x_{i}^{\prime}(t)= & -b_{i}\left(h_{i}^{-1}\left(x_{i}(t)\right)\right) \\
& +\sum_{j=1}^{n}\left[b_{i j}(t) f_{j}\left(h_{j}^{-1}\left(x_{j}\left(t-\tau_{i j}(t)\right)\right)\right)\right. \\
& \left.\quad+c_{i j}(t) \int_{-\infty}^{t} k_{i j}(t-s) g_{j}\left(h_{j}^{-1}\left(x_{j}(s)\right)\right) \mathrm{d} s\right]-I_{i}(t), \quad t \neq t_{k},
\end{aligned}
$$


which can be rewritten as

$$
\begin{aligned}
& x_{i}^{\prime}(t)=-d_{i}\left(x_{i}(t)\right) x_{i}(t)+\sum_{j=1}^{n}[ b_{i j}(t) f_{j}\left(h_{j}^{-1}\left(x_{j}\left(t-\tau_{i j}(t)\right)\right)\right) \\
&\left.\quad+c_{i j}(t) \int_{-\infty}^{t} k_{i j}(t-s) g_{j}\left(h_{j}^{-1}\left(x_{j}(s)\right)\right) \mathrm{d} s\right]-I_{i}(t), \quad t \neq t_{k}, \\
& \Delta x_{i}\left(t_{k}\right)=r_{i}\left(x_{i}\left(t_{k}\right)\right), \quad i=1,2, \ldots, n,
\end{aligned}
$$

where $\left.d_{i}\left(x_{i}(t)\right) \triangleq b_{i}\left(h_{i}^{-1}(z)\right)^{\prime}\right|_{z=\xi_{i}},\left.b_{i}\left(h_{i}^{-1}(z)\right)^{\prime}\right|_{z=\xi_{i}}$ denote the derivative of $b_{i}\left(h_{i}^{-1}(z)\right)$ at point $z=\xi_{i}, z \in R, \xi_{i}$ is between 0 and $x_{i}(t)$.

The existence and global exponential stability of periodic solution for system (2.1) are equivalent to the existence and global exponential stability of periodic solution for system (2.5) or (2.6). So, we investigate the the existence and global exponential stability of periodic solution for system (2.6).

From the definition of $h_{i}^{-1}(u)$, using Lagrange mean value theorem, one gets

$$
\left|h_{i}^{-1}(x)-h_{i}^{-1}(y)\right|=\left|\left(h_{i}^{-1}\right)^{\prime}(\xi)(x-y)\right| \leq \bar{a}_{i}|x-y|, \quad \forall x, y \in R,
$$

where $\xi$ is between $x$ and $y$. Moreover, we have

$$
\begin{aligned}
\left|r_{i}\left(x_{i}\left(t_{k}\right)\right)\right| & \leq \frac{1}{\underline{a}_{i}}\left|\left(1+\gamma_{i k}\right) h_{i}^{-1}\left(x_{i}\left(t_{k}\right)\right)-u_{i}\left(t_{k}\right)\right| \\
& =\frac{\left|\gamma_{i k}\right|}{\underline{a}_{i}}\left|h_{i}^{-1}\left(x_{i}\left(t_{k}\right)\right)\right| \leq \frac{\bar{a}_{i}}{\underline{a}_{i}}\left|\gamma_{i k}\right|\left|x_{i}\left(t_{k}\right)\right| .
\end{aligned}
$$

For convenience, we denote $\tau=\max \left\{\tau_{i j}(t): t \in[0, \omega], i, j=1,2, \ldots, n\right\}, x=$ $\left(x_{1}, x_{2}, \ldots, x_{n}\right)^{T}$ to be a column vector, in which the symbol $\left({ }^{T}\right)$ denotes the transpose of a vector.

Definition 2.1. A function $x \in\left(R, R^{n}\right)$ is said to be a solution of (2.6) if the following conditions are satisfied.

(i) $x(t)$ is absolutely continuous on each $\left(t_{k}, t_{k+1}\right), k \in N$.

(ii) For each $k \in N, x\left(t_{k}^{+}\right)$and $x\left(t_{k}^{-}\right)$exist and $x\left(t_{k}\right)=x\left(t_{k}^{-}\right)$.

(iii) $x(t)$ satisfies (2.6) for almost everywhere and at impulsive points, $t_{k}$ may have discontinuity of the first kind.

The initial condition $\phi=\left(\phi_{1}, \ldots, \phi_{n}\right)^{T}$ of $(2.6)$ is of the form

$$
x_{i}(s)=\phi_{i}(s), \quad s \in(-\infty, 0], i=1, \ldots, n,
$$

where $\phi_{i}(s), i=1,2, \ldots, n$ are continuous functions. 
Definition 2.2. Let $x^{*}(t)$ be an $\omega$-periodic solution of (2.6) with initial value $\phi^{*}=$ $\left(\phi_{1}^{*}, \ldots, \phi_{n}^{*}\right)^{T} \in C\left((-\infty, 0] ; R^{n}\right)$. If there exist constants $\alpha>0, M \geq 1$ such that for every solution $x(t)$ of (2.6) with initial value $\phi \in C\left((-\infty, 0] ; R^{n}\right)$,

$$
\left|x_{i}(t)-x_{i}^{*}(t)\right| \leq M\left\|\phi-\phi^{*}\right\| e^{-\alpha t}, \quad \forall t>0, i=1,2, \ldots, n,
$$

where $\left\|\phi-\phi^{*}\right\|=\sup _{s \leq 0} \max _{1 \leq i \leq n}\left|\phi_{i}(s)-\phi_{i}^{*}(s)\right|$. Then, $x^{*}(t)$ is said to be globally exponentially stable.

Lemma 2.3 (Leray-Schauder). Let $E$ be a Banach space, and let the operator $A: E \rightarrow E$ be completely continuous. If the set $\{\|x\| \mid x \in E, x=\lambda A x, 0<\lambda<1\}$ is bounded, then $A$ has a fixed point in $T$, where

$$
T=\{x \mid x \in E,\|x\| \leq R\}, \quad R=\sup \{\|x\| \mid x=\lambda A x, 0<\lambda<1\} .
$$

\section{Existence of periodic solutions}

Lemma 3.1. Suppose that $\left(H_{1}\right)-\left(H_{5}\right)$ hold and let $x(t)$ be an w-periodic solution of system (2.6). Then,

$$
\begin{aligned}
x_{i}(t)=\int_{0}^{\omega} w_{i}^{x}(t, s)\left[\sum _ { j = 1 } ^ { n } \left[b_{i j}(s) f_{j}\left(h_{j}^{-1}\left(x_{j}\left(s-\tau_{i j}(s)\right)\right)\right)\right.\right. \\
\left.\left.\quad+c_{i j}(s) \int_{-\infty}^{s} k_{i j}(s-v) g_{j}\left(h_{j}^{-1}\left(x_{j}(v)\right)\right) \mathrm{d} v\right]-I_{i}(s)\right] \mathrm{d} s \\
+\sum_{k=1}^{p} w_{i}^{x}\left(t, t_{k}\right) r_{i}\left(x_{i}\left(t_{k}\right)\right), \quad t \in[0, \omega], i=1,2, \ldots, n,
\end{aligned}
$$

where

$$
w_{i}^{x}(t, s)=\frac{1}{1-e^{-\int_{0}^{\omega} d_{i}\left(x_{i}(v)\right) \mathrm{d} v}} \begin{cases}e^{-\int_{s}^{t} d_{i}\left(x_{i}(v)\right) \mathrm{d} v,} & 0 \leq s \leq t \leq \omega, \\ e^{-\left(\int_{0}^{\omega} d_{i}\left(x_{i}(v)\right) d v-\int_{t}^{s} d_{i}\left(x_{i}(v)\right) \mathrm{d} v\right),} & 0 \leq t<s \leq \omega .\end{cases}
$$

Proof. Let $t_{q} \leq t<t_{q+1}, q \leq p$. From the first expression of (2.6), we have

$$
\begin{aligned}
\left(x_{i}(t) e^{\int_{0}^{t} d_{i}\left(x_{i}(s)\right) \mathrm{d} s}\right)^{\prime}=\left\{\sum_{j=1}^{n}[\right. & b_{i j}(t) f_{j}\left(h_{j}^{-1}\left(x_{j}\left(t-\tau_{i j}(t)\right)\right)\right) \\
& \left.\left.\quad+c_{i j}(t) \int_{-\infty}^{t} k_{i j}(t-s) g_{j}\left(h_{j}^{-1}\left(x_{j}(s)\right)\right) \mathrm{d} s\right]-I_{i}(t)\right\} e^{\int_{0}^{t} d_{i}\left(x_{i}(s)\right) \mathrm{d} s} .
\end{aligned}
$$


Integrating (3.3) on intervals $\left(0, t_{1}^{-}\right),\left(t_{1}^{+}, t_{2}^{-}\right), \ldots,\left(t_{q}^{+}, t\right)$ and adding all of them, by the second formula of (2.6), we have

$$
\begin{aligned}
x_{i}(t)= & e^{-\int_{0}^{t} d_{i}\left(x_{i}(s)\right) \mathrm{d} s} x_{i}(0)+\sum_{k=1}^{q} e^{-\int_{t_{k}}^{t} d_{i}\left(x_{i}(s)\right) \mathrm{d} s} r_{i}\left(x_{i}\left(t_{k}\right)\right) \\
& +\int_{0}^{t}\left\{\sum _ { j = 1 } ^ { n } \left[b_{i j}(s) f_{j}\left(h_{j}^{-1}\left(x_{j}\left(s-\tau_{i j}(s)\right)\right)\right)\right.\right. \\
& \left.\left.+c_{i j}(s) \int_{-\infty}^{s} k_{i j}(s-v) g_{j}\left(h_{j}^{-1}\left(x_{j}(v)\right)\right) \mathrm{d} v\right]-I_{i}(s)\right\} e^{-\int_{s}^{t} d_{i}\left(x_{i}(v)\right) \mathrm{d} v} \mathrm{~d} s .
\end{aligned}
$$

Since $x_{i}(\omega)=x_{i}(0)$, from (3.4), we obtain

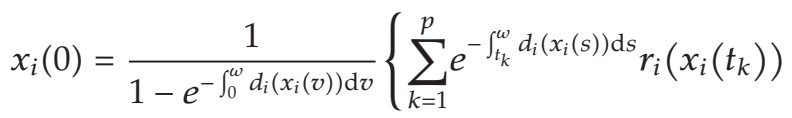

$$
\begin{aligned}
& +\int_{0}^{\omega}\left\{\sum _ { j = 1 } ^ { n } \left[b_{i j}(s) f_{j}\left(h_{j}^{-1}\left(x_{j}\left(s-\tau_{i j}(s)\right)\right)\right)\right.\right. \\
& \left.\left.+c_{i j}(s) \int_{-\infty}^{s} k_{i j}(s-v) g_{j}\left(h_{j}^{-1}\left(x_{j}(v)\right)\right) \mathrm{d} v\right]-I_{i}(s)\right\} \\
& \left.\times e^{-\int_{s}^{\omega} d_{i}\left(x_{i}(v)\right) \mathrm{d} v} \mathrm{~d} s\right\} .
\end{aligned}
$$

Substituting (3.5) into (3.4), we obtain (3.1). This completes the proof.

In order to use Lemma 2.3, we take $P C\left(J, R^{n}\right)=\left\{x(t) \mid x: J=[0, \omega] \rightarrow R^{n}\right.$ as piecewise continuous periodic solution at $t \neq t_{k}$, and $x\left(t_{k}^{+}\right), x\left(t_{k}^{-}\right)=x\left(t_{k}\right)$ exist at $t=t_{k}, k=$ $1,2, \ldots, p\}$. Then, $P C\left(J, R^{n}\right)$ is a Banach space with the norm

$$
\|x\|=\max _{1 \leq i \leq n}\left\{\left|x_{i}\right|_{0}\right\}, \quad\left|x_{i}\right|_{0}=\sup _{0 \leq t \leq \omega}\left|x_{i}(t)\right|, \quad i=1,2, \ldots, n .
$$

Set a mapping $\Phi: P C\left(J, R^{n}\right) \rightarrow P C\left(J, R^{n}\right)$ by setting

$$
(\Phi x)(t)=x(t), \quad t \in J=[0, \omega]
$$

where

$$
\begin{aligned}
(\Phi x)_{i}(t)=\int_{0}^{\omega} w_{i}^{x}(t, s)\left[\sum _ { j = 1 } ^ { n } \left[b_{i j}(s) f_{j}\left(h_{j}^{-1}\left(x_{j}\left(s-\tau_{i j}(s)\right)\right)\right)\right.\right. & \\
& \left.\left.\quad+c_{i j}(s) \int_{-\infty}^{s} k_{i j}(s-v) g_{j}\left(h_{j}^{-1}\left(x_{j}(v)\right)\right) \mathrm{d} v\right]-I_{i}(s)\right] \mathrm{d} s \\
& +\sum_{k=1}^{p} w_{i}^{x}\left(t, t_{k}\right) r_{i}\left(x_{i}\left(t_{k}\right)\right) .
\end{aligned}
$$


It is easy to know the fact that the existence of $\omega$-periodic solution of (2.6) is equivalent to the existence of fixed point of the mapping $\Phi$ in $P C\left(J, R^{n}\right)$.

Lemma 3.2. Suppose that $\left(H_{1}\right)-\left(H_{7}\right)$ hold. Then, $\Phi: P C\left(J, R^{n}\right) \rightarrow P C\left(J, R^{n}\right)$ is completely continuous.

Proof. First, we show that $\Phi: P C\left(J, R^{n}\right) \rightarrow P C\left(J, R^{n}\right)$ is continuous. For any $\varepsilon>0$, we take $0<\delta<\varepsilon / \max _{1 \leq i \leq n}\left\{\theta+\sum_{k=1}^{p} \eta_{i k} /\left(1-e^{-q_{i} \omega}\right)\right\}$, where $\eta_{i k}=\left(\bar{a}_{i} / \underline{a}_{i}\right)\left|1+\gamma_{i k}\right|+1$. Then, for all $x, y \in P C\left(J, R^{n}\right)$ and $\|x-y\|<\delta$, we have

$$
\begin{gathered}
\|\Phi x-\Phi y\|=\max _{1 \leq i \leq n} \sup _{0 \leq t \leq \omega}\left\{\mid \int_{0}^{\omega} w_{i}^{x}(t, s)\right. \\
\quad \times \sum_{j=1}^{n}\left[b _ { i j } ( s ) \left[f_{j}\left(h_{j}^{-1}\left(x_{j}\left(s-\tau_{i j}(s)\right)\right)\right)-f_{j}\left(h_{j}^{-1}\left(y_{j}\left(s-\tau_{i j}(s)\right)\right)\right]\right.\right. \\
\left.\quad+c_{i j}(s) \int_{-\infty}^{s} k_{i j}(s-v)\left[g_{j}\left(h_{j}^{-1}\left(x_{j}(v)\right)\right)-g_{j}\left(h_{j}^{-1}\left(y_{j}(v)\right)\right)\right] \mathrm{d} v\right] \mathrm{d} s \\
\left.\quad+\sum_{k=1}^{p} w_{i}^{x}\left(t, t_{k}\right)\left[r_{i}\left(x_{i}\left(t_{k}\right)\right)-r_{i}\left(y_{i}\left(t_{k}\right)\right)\right] \mid\right\} \\
\leq \max _{1 \leq i \leq n} \sup _{0 \leq t \leq \omega}\left\{\int_{0}^{\omega} w_{i}^{x}(t, s) q_{i} \theta \mathrm{d} s+\sum_{k=1}^{p} \frac{\eta_{i k}}{1-e^{-q_{i} \omega}}\right\}\|x-y\| \\
<\max _{1 \leq i \leq n}\left\{\theta+\frac{\sum_{k=1}^{p} \eta_{i k}}{\left.1-e^{-q_{i} \omega}\right\}}\right\}<\varepsilon .
\end{gathered}
$$

Hence, $\Phi$ is continuous.

Next, we show $\Phi$ maps bounded set into bounded set. For any $x \in P C\left(J, R^{n}\right)$ with $\|x\|<D$, where $D$ is some positive constant, we have

$$
\begin{gathered}
\|\Phi x\|=\max _{1 \leq i \leq n} \sup _{0 \leq t \leq \omega}\left\{\mid \int_{0}^{\omega} w_{i}^{x}(t, s)\right. \\
\times\left[\sum _ { j = 1 } ^ { n } \left[b_{i j}(s) f_{j}\left(h_{j}^{-1}\left(x_{j}\left(s-\tau_{i j}(s)\right)\right)\right)\right.\right. \\
\left.\left.+c_{i j}(s) \int_{-\infty}^{s} k_{i j}(s-v) g_{j}\left(h_{j}^{-1}\left(x_{j}(v)\right)\right) \mathrm{d} v\right]-I_{i}(s)\right] \mathrm{d} s \\
<\max _{1 \leq i \leq n}\left\{\theta+\frac{\left.\sum_{k=1}^{p} w_{i}^{x}\left(t, t_{k}\right) r_{i}\left(x_{i}\left(t_{k}\right)\right) \mid\right\}}{\underline{a}_{i}\left(1-e^{-q_{i} \omega}\right)} \sum_{k=1}^{p}\left|r_{i k}\right|\right\} D \\
+\max _{1 \leq i \leq n}\left\{\frac{1}{q_{i}}\left[\sum_{j=1}^{n}\left[\bar{b}_{i j}\left|f_{j}(0)\right|+\bar{c}_{i j} \beta_{i j}\left|g_{j}(0)\right|\right]+\bar{I}_{i}\right]\right\},
\end{gathered}
$$


where $\bar{b}_{i j}=\max _{t \in[0, \omega]}\left|b_{i j}(t)\right|, \bar{c}_{i j}=\max _{t \in[0, \omega]}\left|c_{i j}(t)\right|, \bar{I}_{i}=\max _{t \in[0, \omega]}\left|I_{i}(t)\right|$. Equation (3.10) implies that $\|\Phi x\|$ is uniformly bounded for any $\|x\|<D$. Hence, $\left\{\Phi x \mid x \in P C\left(J, R^{n}\right)\right\}$ is a family of uniformly bounded and equicontinuous functions on $R$. By using the Arzela-Ascoli theorem, $\Phi: P C\left(J, R^{n}\right) \rightarrow P C\left(J, R^{n}\right)$ is compact. Therefore, $\Phi: P C\left(J, R^{n}\right) \rightarrow P C\left(J, R^{n}\right)$ is completely continuous. This completes the proof.

Theorem 3.3. Suppose that $\left(H_{1}\right)-\left(H_{7}\right)$ hold. Then, system (2.6) has an w-periodic solution.

Proof. Let $x \in P C\left(J, R^{n}\right), t \in J$. We consider the operator equation

$$
x=\lambda \Phi x, \quad \lambda \in(0,1) .
$$

If $x$ is a solution of (3.11), for $t \in J$, we obtain

$$
\begin{aligned}
\|x\| \leq\|\Phi x\| \leq & \max _{1 \leq i \leq n}\left\{\theta+\frac{\bar{a}_{i}}{\underline{a}_{i}\left(1-e^{-q_{i} \omega}\right)} \sum_{k=1}^{p}\left|\gamma_{i k}\right|\right\}\|x\| \\
& +\max _{1 \leq i \leq n}\left\{\frac{1}{q_{i}}\left[\sum_{j=1}^{n}\left[\bar{b}_{i j}\left|f_{j}(0)\right|+\bar{c}_{i j} \beta_{i j}\left|g_{j}(0)\right|\right]+\bar{I}_{i}\right]\right\},
\end{aligned}
$$

where this and $\max _{1 \leq i \leq n}\left\{\theta+\left(\bar{a}_{i} / \underline{a}_{i}\left(1-e^{-q_{i} \omega}\right)\right) \sum_{k=1}^{p}\left|\gamma_{i k}\right|\right\}<1$ imply that

$$
\|x\| \leq \frac{\max _{1 \leq i \leq n}\left\{\left(1 / q_{i}\right)\left[\sum_{j=1}^{n}\left[\bar{b}_{i j}\left|f_{j}(0)\right|+\bar{c}_{i j} \beta_{i j}\left|g_{j}(0)\right|\right]+\bar{I}_{i}\right]\right\}}{1-\max _{1 \leq i \leq n}\left\{\theta+\left(\bar{a}_{i} / \underline{a}_{i}\left(1-e^{-q_{i} \omega}\right)\right) \sum_{k=1}^{p}\left|\gamma_{i k}\right|\right\}} \triangleq R .
$$

This shows that $\|x\|$ of (3.11) is bounded, which is independent of $\lambda \in(0,1)$. In view of Lemma 2.3, we obtain that $\Phi$ has a fixed point. Hence, system (2.6) has one w-periodic solution with $\|x\| \leq R$. This completes the proof.

\section{Global exponential stability of periodic solution}

In this section, we will construct some suitable Lyapunov functionals to derive sufficient conditions which ensure that (2.6) has a unique $\omega$-periodic solution, and all solutions of (2.6) exponentially converge to its unique $\omega$-periodic solution.

Theorem 4.1. Assume that $\left(H_{1}\right)-\left(H_{8}\right)$ hold and

$\left(\mathrm{H}_{9}\right) \ln M_{k} /\left(t_{k}-t_{k-1}\right) \leq \eta<\alpha$, where $M_{k}=\max _{1 \leq i \leq n}\left\{\left(\bar{a}_{i} / \underline{a}_{i}\right)\left|1+\gamma_{i k}\right|, 1\right\}, \eta$ is a positive constant, $\alpha$ is given in the proof of this theorem, $t_{0}=t_{p}-\omega$.

Then, system (2.6) has exactly one w-periodic solution, which is globally exponentially stable with the convergence index $\alpha-\eta$.

Proof. By Theorem 3.3, there exists an $\omega$-periodic solution $x^{*}(t)=\left(x_{1}^{*}(t), x_{2}^{*}(t), \ldots, x_{n}^{*}(t)\right)^{T}$ of (2.6) with initial value $\phi^{*}=\left(\phi_{1}^{*}, \ldots, \phi_{n}^{*}\right)^{T}$. Suppose that $x(t)$ is an arbitrary solution of system 
(2.6) with initial value $\phi=\left(\phi_{1}, \ldots, \phi_{n}\right)^{T}$. Set $z(t)=\left(z_{1}(t), z_{2}(t), \ldots, z_{n}(t)\right)^{T}=x(t)-x^{*}(t)$, then, from system (2.5), we have

$$
\begin{aligned}
z_{i}^{\prime}(t)=- & {\left[b_{i}\left(h_{i}^{-1}\left(x_{i}(t)\right)\right)-b_{i}\left(h_{i}^{-1}\left(x_{i}^{*}(t)\right)\right)\right] } \\
+ & +\sum_{j=1}^{n}\left[b_{i j}(t)\left[f_{j}\left(h_{j}^{-1}\left(x_{j}\left(t-\tau_{i j}(t)\right)\right)\right)-f_{j}\left(h_{j}^{-1}\left(x_{j}^{*}\left(t-\tau_{i j}(t)\right)\right)\right)\right]\right. \\
& \left.\quad+c_{i j}(t) \int_{-\infty}^{t} k_{i j}(t-s)\left[g_{j}\left(h_{j}^{-1}\left(x_{j}(s)\right)\right)-g_{j}\left(h_{j}^{-1}\left(x_{j}^{*}(s)\right)\right)\right] \mathrm{d} s\right], \quad t \neq t_{k}, \\
z_{i}\left(t_{k}^{+}\right)= & h_{i}\left(\left(1+b_{i k}\right) h_{i}^{-1}\left(x_{i}\left(t_{k}\right)\right)\right)-h_{i}\left(\left(1+b_{i k}\right) h_{i}^{-1}\left(x_{i}^{*}\left(t_{k}\right)\right)\right), \quad i=1,2, \ldots, n .
\end{aligned}
$$

By $\theta<1$, we have

$$
-q_{i}+\sum_{j=1}^{n} \bar{a}_{j}\left[\left|b_{i j}(t)\right| L_{j}^{f}+\left|c_{i j}(t)\right| L_{j}^{g} \beta_{i j}\right]<0, \quad i=1,2, \ldots, n .
$$

Set

$$
h_{i}(\lambda)=\lambda-q_{i}+\sum_{j=1}^{n} \bar{a}_{j}\left[\left|b_{i j}(t)\right| L_{j}^{f} e^{\lambda \tau}+\left|c_{i j}(t)\right| L_{j}^{g} \int_{0}^{+\infty}\left|k_{i j}(s)\right| e^{\lambda s} \mathrm{~d} s\right], \quad i=1,2, \ldots, n
$$

Clearly, $h_{i}(\lambda), i=1,2, \ldots, n$ are continuous functions on $R$. Since

$$
\begin{gathered}
h_{i}(0) \leq-q_{i}+\sum_{j=1}^{n} \bar{a}_{j}\left[\left|b_{i j}(t)\right| L_{j}^{f}+\left|c_{i j}(t)\right| L_{j}^{g} \beta_{i j}\right]<0, \\
\frac{\mathrm{d} h_{i}(\lambda)}{\mathrm{d} \lambda}=1+\sum_{j=1}^{n} \bar{a}_{j}\left[\tau\left|b_{i j}(t)\right| L_{j}^{f} e^{\lambda \tau}+\left|c_{i j}(t)\right| L_{j}^{g} \int_{0}^{+\infty}\left|k_{i j}(s)\right| s e^{\lambda s} \mathrm{~d} s\right]>0,
\end{gathered}
$$

and $h_{i}(+\infty)=+\infty$, hence $h_{i}(\lambda), i=1,2, \ldots, n$ are strictly monotone increasing functions. Therefore, for any $i \in\{1,2, \ldots, n\}$ and $t \geq 0$, there is a unique $\lambda(t)$ such that

$$
\lambda(t)-q_{i}+\sum_{j=1}^{n} \bar{a}_{j}\left[\left|b_{i j}(t)\right| L_{j}^{f} e^{\lambda(t) \tau}+\left|c_{i j}(t)\right| L_{j}^{g} \int_{0}^{+\infty}\left|k_{i j}(s)\right| e^{\lambda(t) s} \mathrm{~d} s\right]=0
$$

Let $\lambda_{i}^{*}=\inf _{t \geq 0}\left\{\lambda(t) \mid \lambda(t)-q_{i}+\sum_{j=1}^{n} \bar{a}_{j}\left[\left|b_{i j}(t)\right| L_{j}^{f} e^{\lambda(t) \tau}+\left|c_{i j}(t)\right| L_{j}^{g} \int_{0}^{+\infty}\left|k_{i j}(s)\right| e^{\lambda(t) s} \mathrm{~d} s\right]=0\right\}$. Obviously, $\lambda_{i}^{*} \geq 0, i=1,2, \ldots, n$. Now, we will prove that $\lambda_{i}^{*}>0, i=1,2, \ldots, n$. Suppose this is not true, from (4.2), there exists a positive constant $\eta$ such that

$$
\inf _{t \geq 0,1 \leq i \leq n}\left\{\frac{q_{i}-\sum_{j=1}^{n} \bar{a}_{j}\left[\left|b_{i j}(t)\right| L_{j}^{f}+\left|c_{i j}(t)\right| L_{j}^{g} \beta_{i j}\right]}{1+\sum_{j=1}^{n} \bar{a}_{j}\left[1.5 \tau\left|b_{i j}(t)\right| L_{j}^{f}+\left|c_{i j}(t)\right| L_{j}^{g} \beta_{i j}\right]}\right\} \geq \eta .
$$


Pick small $\varepsilon>0$, then there exists $t^{i} \geq 0$ such that

$$
0<\lambda_{i}^{*}\left(t^{i}\right)<\varepsilon<\eta, \quad \int_{0}^{+\infty}\left|k_{i j}(s)\right| e^{\varepsilon s} \mathrm{~d} s<\beta_{i j}+\eta .
$$

Let us recall the inequality $e^{x}<1+1.5 x$ for sufficiently small $x>0$, then, we obtain

$$
\begin{aligned}
0= & \lambda_{i}^{*}\left(t^{i}\right)-q_{i}+\sum_{j=1}^{n} \bar{a}_{j}\left[\left|b_{i j}\left(t^{i}\right)\right| L_{j}^{f} e^{\lambda_{i}^{*}\left(t^{i}\right) \tau}+\left|c_{i j}\left(t^{i}\right)\right| L_{j}^{g} \int_{0}^{+\infty}\left|k_{i j}(s)\right| e^{\lambda_{i}^{*}\left(t^{i}\right) s} \mathrm{~d} s\right] \\
< & \eta-q_{i}+\sum_{j=1}^{n} \bar{a}_{j}\left[\left|b_{i j}\left(t^{i}\right)\right| L_{j}^{f}(1+1.5 \eta \tau)+\left|c_{i j}\left(t^{i}\right)\right| L_{j}^{g}\left(\beta_{i j}+\eta\right)\right] \\
\leq & -q_{i}+\sum_{j=1}^{n} \bar{a}_{j}\left[\left|b_{i j}\left(t^{i}\right)\right| L_{j}^{f}+\left|c_{i j}\left(t^{i}\right)\right| L_{j}^{g} \beta_{i j}\right] \\
& +\frac{q_{i}-\sum_{j=1}^{n} \bar{a}_{j}\left[\left|b_{i j}\left(t^{i}\right)\right| L_{j}^{f}+\left|c_{i j}\left(t^{i}\right)\right| L_{j}^{g} \beta_{i j}\right]}{1+\sum_{j=1}^{n} \bar{a}_{j}\left[1.5 \tau\left|b_{i j}\left(t^{i}\right)\right| L_{j}^{f}+\left|c_{i j}\left(t^{i}\right)\right| L_{j}^{g} \beta_{i j}\right]} \\
& \times\left\{1+\sum_{j=1}^{n} \bar{a}_{j}\left[1.5 \tau\left|b_{i j}\left(t^{i}\right)\right| L_{j}^{f}+\left|c_{i j}\left(t^{i}\right)\right| L_{j}^{g} \beta_{i j}\right]\right\}=0,
\end{aligned}
$$

which is a contradiction, and hence, $\lambda_{i}^{*}>0, i=1,2, \ldots, n$.

Let $\alpha=\min \left\{\lambda_{1}^{*}, \lambda_{2}^{*}, \ldots, \lambda_{n}^{*}\right\}$. Obviously, for all $t \geq 0$, we have

$$
h_{i}(\alpha)=\alpha-q_{i}+\sum_{j=1}^{n} \bar{a}_{j}\left[\left|b_{i j}(t)\right| L_{j}^{f} e^{\alpha \tau}+\left|c_{i j}(t)\right| L_{j}^{g} \int_{0}^{+\infty}\left|k_{i j}(s)\right| e^{\alpha s} \mathrm{~d} s\right] \leq 0, \quad i=1,2, \ldots, n .
$$

It is obvious that

$$
\left|z_{i}(t)\right| \leq\left\|\phi-\phi^{*}\right\| \leq\left\|\phi-\phi^{*}\right\| e^{-\alpha t}, \quad \text { for } t \in(-\infty, 0], i=1,2, \ldots, n,
$$

where $\left\|\phi-\phi^{*}\right\|$ is defined as that in Definition 2.2.

Define the Lyapunov functional $V=\left(V_{1}, V_{2}, \ldots, V_{n}\right)^{T}$ by $V_{i}=e^{\alpha t}\left|z_{i}(t)\right|, i=1,2, \ldots, n$. In view of (4.1), for $t \neq t_{k}, k=1,2, \ldots$, we obtain

$$
\begin{aligned}
& \frac{\mathrm{d}^{+} V_{i}(t) \leq e^{\alpha t}\left\{\left(\alpha-q_{i}\right)\left|z_{i}(t)\right|+\sum_{j=1}^{n}\right.}{\mathrm{d} t}|| b_{i j}(t)\left|L_{j}^{f} \bar{a}_{j}\right| z_{j}\left(t-\tau_{i j}(t)\right) \mid \\
&\left.\left.+\left|c_{i j}(t)\right| \int_{-\infty}^{t}\left|k_{i j}(t-s)\right| L_{j}^{g} \bar{a}_{j}\left|z_{j}(s)\right| \mathrm{d} s\right]\right\} \\
& \leq\left(\alpha-q_{i}\right) V_{i}(t)+\sum_{j=1}^{n} \bar{a}_{j}\left[\left|b_{i j}(t)\right| e^{\alpha \tau} L_{j}^{f} V_{j}\left(t-\tau_{i j}(t)\right)\right. \\
&\left.+\left|c_{i j}(t)\right| L_{j}^{g} \int_{-\infty}^{t}\left|k_{i j}(t-s)\right| e^{\alpha(t-s)} V_{j}(s) \mathrm{d} s\right] .
\end{aligned}
$$


We claim that

$$
V_{i}(t)=\left|z_{i}(t)\right| e^{\alpha t} \leq\left\|\phi-\phi^{*}\right\|, \quad i=1,2, \ldots, n, \forall t \in\left[0, t_{1}\right) .
$$

Contrarily, there must exist $i_{0} \in\{1,2, \ldots, n\}$ and $0<\tilde{t}<t_{1}$ such that

$$
V_{i_{0}}(\tilde{t})=\left\|\phi-\phi^{*}\right\|, \quad \frac{\mathrm{d}^{+} V_{i_{0}}(\tilde{t})}{\mathrm{d} t}>0, \quad V_{i}(t) \leq\left\|\phi-\phi^{*}\right\|, \quad \forall t \in(-\infty, \tilde{t}], i=1,2, \ldots, n .
$$

Together with (4.11) and (4.13), we obtain

$$
\begin{aligned}
0 & <\frac{\mathrm{d}^{+} V_{i_{0}}(\tilde{t})}{\mathrm{d} t} \\
& \leq\left\|\phi-\phi^{*}\right\|\left\{\alpha-q_{i_{0}}+\sum_{j=1}^{n} \bar{a}_{j}\left[\left|b_{i_{0} j}(\tilde{t})\right| e^{\alpha \tau} L_{j}^{f}+\left|c_{i_{0} j}(\tilde{t})\right| L_{j}^{g} \int_{0}^{+\infty}\left|k_{i_{0} j}(s)\right| e^{\alpha s} \mathrm{~d} s\right]\right\} .
\end{aligned}
$$

Hence,

$$
0<\alpha-q_{i_{0}}+\sum_{j=1}^{n} \bar{a}_{j}\left[\left|b_{i_{0} j}(\tilde{t})\right| e^{\alpha \tau} L_{j}^{f}+\left|c_{i_{0} j}(\tilde{t})\right| L_{j}^{g} \int_{0}^{+\infty}\left|k_{i_{0} j}(s)\right| e^{\alpha s} \mathrm{~d} s\right]
$$

which contradicts (4.9). Hence, (4.12) holds. It follows that

$$
\left|z_{i}(t)\right| \leq\left\|\phi-\phi^{*}\right\| e^{-\alpha t}, \quad \forall t \in\left[0, t_{1}\right), i=1,2, \ldots, n .
$$

When $t=t_{1}$, from the second expression of (4.1), we have

$$
\begin{aligned}
\left|z_{i}\left(t_{1}^{+}\right)\right| & \leq \frac{\bar{a}_{i}}{\underline{a}_{i}}\left|1+\gamma_{i 1}\right|\left|z_{i}\left(t_{1}\right)\right| \leq M_{1} \lim _{t \rightarrow t_{1}^{-}}\left|z_{i}\left(t_{1}\right)\right| \\
& \leq M_{1}\left\|\phi-\phi^{*}\right\| e^{-\alpha t_{1}}, \quad i=1,2, \ldots, n .
\end{aligned}
$$

Similar to the steps of (4.9)-(4.16), we can also prove that

$$
\left|z_{i}(t)\right| \leq M_{1}\left\|\phi-\phi^{*}\right\| e^{-\alpha t}, \quad t \in\left[t_{1}, t_{2}\right), i=1,2, \ldots, n .
$$

When $t=t_{2}$, again, from the second expression of (4.1), we have

$$
\left|z_{i}\left(t_{2}^{+}\right)\right| \leq \frac{\bar{a}_{i}}{\underline{a}_{i}}\left|1+b_{i 2}\right|\left|z_{i}\left(t_{2}\right)\right| \leq M_{1} M_{2}\left\|\phi-\phi^{*}\right\| e^{-\alpha t_{2}}, \quad i=1,2, \ldots, n .
$$

By repeating the same procedure, we can deduce the following general result:

$$
\left|z_{i}(t)\right| \leq M_{1} M_{2} \cdots M_{k}\left\|\phi-\phi^{*}\right\| e^{-\alpha t}, \quad t \in\left[t_{k}, t_{k+1}\right), i=1,2, \ldots, n .
$$


By $\left(\mathrm{H}_{9}\right)$, we have $M_{k} \leq e^{\eta\left(t_{k}-t_{k-1}\right)}$, which implies that

$$
M_{1} M_{2} \cdots M_{k} \leq e^{\eta\left(t_{1}-t_{0}\right)} e^{\eta\left(t_{2}-t_{1}\right)}, \ldots, e^{\eta\left(t_{k}-t_{k-1}\right)} \leq e^{\eta\left(\omega-t_{p}\right)} e^{\eta t}, \quad t \in\left[t_{k}, t_{k+1}\right) .
$$

So, in light of (4.20) and (4.21), we have

$$
\left|x_{i}(t)-x_{i}^{*}(t)\right|=\left|z_{i}(t)\right| \leq e^{\eta\left(\omega-t_{p}\right)}\left\|\phi-\phi^{*}\right\| e^{-(\alpha-\eta) t}, \quad \forall t>0, i=1,2, \ldots, n .
$$

In view of Definition 2.2, the $\omega$-periodic solution $x^{*}(t)$ of system (2.6) is globally exponentially stable with the convergence index $\alpha-\eta$. This completes the proof.

Remark 4.2. Note that we have dropped the restriction: the activation functions are bounded and $\left|1+\gamma_{i k}\right| \leq 1$, which is indispensable in $[13,17]$.

Remark 4.3. To the best of our knowledge, most of the existing research papers only give the existence of the convergence index, while we offer the precise convergence index in Theorem 4.1.

\section{Application}

In this section, we give an example to illustrate that our results are feasible. Consider the following Cohen-Grossberg neural networks with mixed delays and impulses:

$$
\begin{gathered}
\begin{aligned}
u_{i}^{\prime}(t)=-a_{i}\left(u_{i}(t)\right)\left\{b_{i}\left(u_{i}(t)\right)-\sum_{j=1}^{2}[\right. & b_{i j}(t) f_{j}\left(u_{j}\left(t-\tau_{i j}(t)\right)\right) \\
& \left.\left.+c_{i j}(t) \int_{-\infty}^{t} k_{i j}(t-s) g_{j}\left(u_{j}(s)\right) \mathrm{d} s\right]+I_{i}(t)\right\}, \quad t \neq t_{k},
\end{aligned} \\
\Delta u_{i}\left(t_{k}\right)=u_{i}\left(t_{k}^{+}\right)-u_{i}\left(t_{k}^{-}\right)=0.02 u_{i}\left(t_{k}\right), \quad i=1,2, t_{k}=k-0.5, k=1,2, \ldots,
\end{gathered}
$$

where $f_{1}(u)=f_{2}(u)=g_{1}(u)=g_{2}(u)=|u+1|, a_{1}(u)=3+\sin u, a_{2}(u)=3-\cos u, b_{1}(u)=$ $5 u+\sin u, b_{2}(u)=5 u-\cos u, I_{i}(t)=\sin \pi t, \tau_{i j}(t)=(\cos \pi t)^{2}, k_{i j}=\sin (t-s) e^{-2(t-s)}, i, j=1,2$ :

$$
\left(\begin{array}{ll}
b_{11} & b_{12} \\
b_{21} & b_{22}
\end{array}\right)=\left(\begin{array}{ll}
\frac{1}{6} \sin \frac{\pi t}{2} & \frac{1}{6} \cos \frac{\pi t}{2} \\
\frac{1}{10} \sin \frac{\pi t}{2} & \frac{1}{8} \cos \frac{\pi t}{2}
\end{array}\right), \quad\left(\begin{array}{ll}
c_{11} & c_{12} \\
c_{21} & c_{22}
\end{array}\right)=\left(\begin{array}{lll}
\frac{1}{6} \cos \frac{\pi t}{2} & \frac{1}{6} \sin \frac{\pi t}{2} \\
\frac{1}{10} \sin \frac{\pi t}{2} & \frac{1}{12} \cos \frac{\pi t}{2}
\end{array}\right) .
$$

We have $\omega=4, L_{1}^{f}=L_{2}^{f}=L_{1}^{g}=L_{2}^{g}=1, \bar{a}_{1}=\bar{a}_{2}=4, \underline{a}_{1}=\underline{a}_{2}=2, l_{1}=l_{2}=4, \beta_{i j}=1 / 2$ :

$$
\theta \approx 0.1863<1, \quad \max _{1 \leq i \leq 2}\left\{\theta+\frac{\bar{a}_{i}}{\underline{a}_{i}\left(1-e^{-q_{i} \omega}\right)} \sum_{k=1}^{p}\left|b_{i k}\right|\right\} \approx 0.3463<1
$$




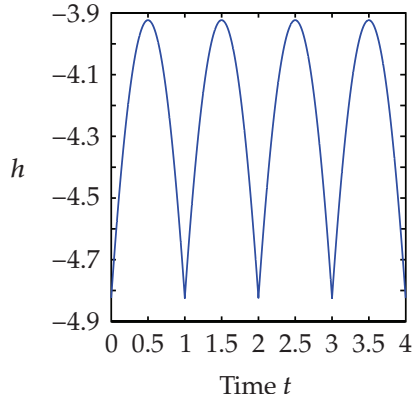

(a)

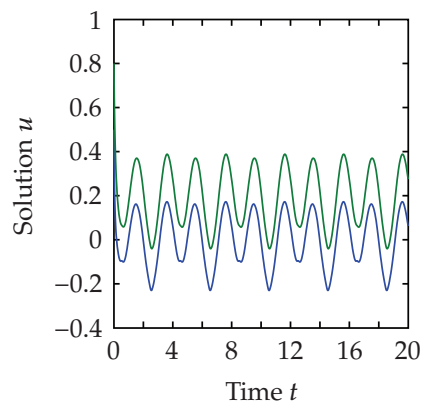

(d)

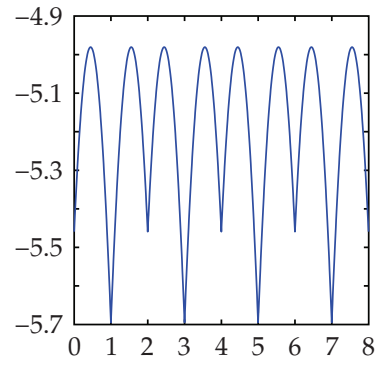

(b)

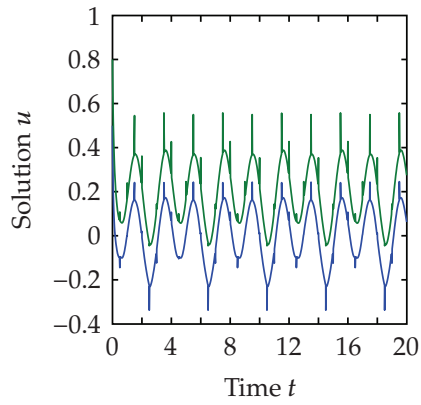

(e)

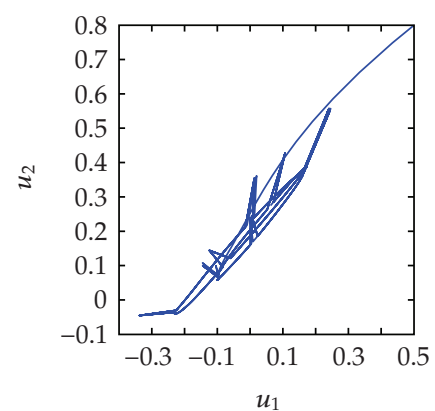

(g)

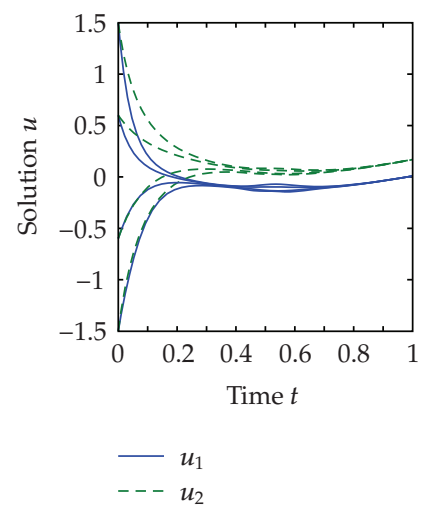

(c)

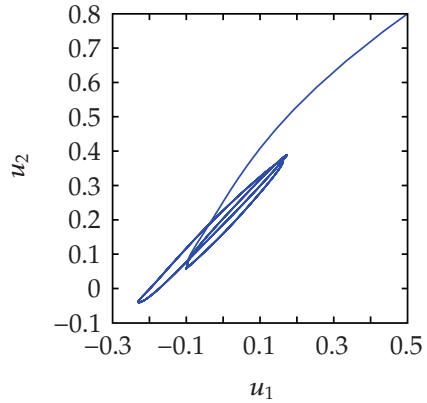

(f) Figure 1: (a) The trajectory of $h_{1}(\lambda)$. (b) The trajectory of $h_{2}(\lambda)$. (c) Numeric simulation of $\left(u_{1}, u_{2}\right)$. (d)
Numeric simulation of $\left(u_{1}, u_{2}\right)$ without impulses. (e) Numeric simulation of $\left(u_{1}, u_{2}\right)$ with impulses. (f) Numeric simulation of $\left(u_{1}, u_{2}\right)$ without impulses. (g) Numeric simulation of $\left(u_{1}, u_{2}\right)$ with impulses.

$\ln M_{k} /\left(t_{k}-t_{k-1}\right)=\ln 2.04=0.7129<1$. Submitting $\lambda=1$ into (4.9), we have $h_{1}(\lambda)<-3.9<0$, $h_{2}(\lambda)<-4.9<0$, (see Figures $1(\mathrm{a})$ and $1(\mathrm{~b})$ ). This implies that $\ln 2.04<1<\alpha$. Hence, all the conditions needed in Theorem 4.1 are satisfied. Therefore, system (5.1) has a unique 4periodic solution, which is globally exponentially stable (see Figures $1(\mathrm{c})-1(\mathrm{~g})$ ).

Remark 5.1. System (5.1) is a simple CGNNs with time-varying coefficients, mixed delays, and impulses. In system (5.1), the activation functions are all unbounded and 
$\left|1+b_{i k}\right|=1.02>1, i=1,2$. Thus, none of the results in [13-17] can be applied to (5.1). This implies that the results of this paper are new and complement previously known results.

\section{Acknowledgments}

The authors would like to thank the reviewers for their valuable comments and constructive suggestions which considerably improve the presentation of this paper. This work was supported in part by the China Postdoctoral Science Foundation (20070410300, 200801336), the Foundation of Chinese Society for Electrical Engineering, the Hunan Provincial Natural Science Foundation of China (07JJ4001), the Scientific Research Fund of Yunnan Provincial Education Department (07Y10085), the Key Scientific Research Fund of Yunnan Provincial Education Department (5Z0071A), and the Scientific Research Fund of Honghe University (XSS07001).

\section{References}

[1] M. A. Cohen and S. Grossberg, "Absolute stability of global pattern formation and parallel memory storage by competitive neural networks," IEEE Transactions on Systems, Man, and Cybernetics, vol. 13, no. 5, pp. 815-826, 1983.

[2] C. Huang and L. Huang, "Dynamics of a class of Cohen-Grossberg neural networks with timevarying delays," Nonlinear Analysis: Real World Applications, vol. 8, no. 1, pp. 40-52, 2007.

[3] T. Chen and L. Rong, "Robust global exponential stability of Cohen-Grossberg neural networks with time delays," IEEE Transactions on Neural Networks, vol. 15, no. 1, pp. 203-206, 2004.

[4] J. Cao and J. Liang, "Boundedness and stability for Cohen-Grossberg neural network with timevarying delays," Journal of Mathematical Analysis and Applications, vol. 296, no. 2, pp. 665-685, 2004.

[5] W. Zhao, "Global exponential stability analysis of Cohen-Grossberg neural network with delays," Communications in Nonlinear Science and Numerical Simulation, vol. 13, no. 5, pp. 847-856, 2008.

[6] K. Yuan and J. Cao, "An analysis of global asymptotic stability of delayed Cohen-Grossberg neural networks via nonsmooth analysis," IEEE Transactions on Circuits and Systems I, vol. 52, no. 9, pp. 18541861, 2005.

[7] L. Huang, C. Huang, and B. Liu, "Dynamics of a class of cellular neural networks with time-varying delays," Physics Letters A, vol. 345, no. 4-6, pp. 330-344, 2005.

[8] J. Zhang, Y. Suda, and H. Komine, "Global exponential stability of Cohen-Grossberg neural networks with variable delays," Physics Letters A, vol. 338, no. 1, pp. 44-50, 2005.

[9] W. Lu and T. Chen, "New conditions on global stability of Cohen-Grossberg neural networks," Neural Computation, vol. 15, no. 5, pp. 1173-1189, 2003.

[10] T. Chen and L. Rong, "Delay-independent stability analysis of Cohen-Grossberg neural networks," Physics Letters A, vol. 317, no. 5-6, pp. 436-449, 2003.

[11] L. Wang and X. Zou, "Exponential stability of Cohen-Grossberg neural networks," Neural Networks, vol. 15, no. 3, pp. 415-422, 2002.

[12] L. Wan and J. Sun, "Global asymptotic stability of Cohen-Grossberg neural network with continuously distributed delays," Physics Letters A, vol. 342, no. 4, pp. 331-340, 2005.

[13] Z. Chen and J. Ruan, "Global stability analysis of impulsive Cohen-Grossberg neural networks with delay," Physics Letters A, vol. 345, no. 1-3, pp. 101-111, 2005.

[14] Q. Song and J. Zhang, "Global exponential stability of impulsive Cohen-Grossberg neural network with time-varying delays," Nonlinear Analysis: Real World Applications, vol. 9, no. 2, pp. 500-510, 2008.

[15] Q. Song and J. Cao, "Impulsive effects on stability of fuzzy Cohen-Grossberg neural networks with time-varying delays," IEEE Transactions on Systems, Man, and Cybernetics, Part B, vol. 37, no. 3, pp. 733-741, 2007.

[16] Q. Song and Z. Wang, "Stability analysis of impulsive stochastic Cohen-Grossberg neural networks with mixed time delays," Physica A, vol. 387, no. 13, pp. 3314-3326, 2008.

[17] C. Bai, "Stability analysis of Cohen-Grossberg BAM neural networks with delays and impulses," Chaos, Solitons \& Fractals, vol. 35, no. 2, pp. 263-267, 2008. 


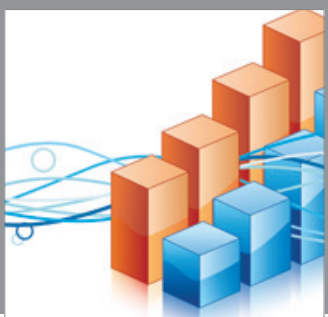

Advances in

Operations Research

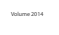

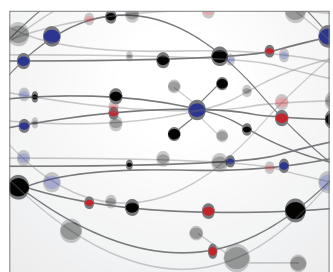

\section{The Scientific} World Journal
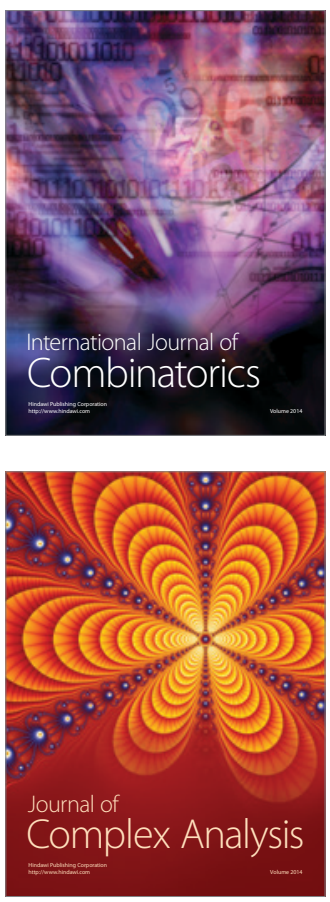

International Journal of

Mathematics and

Mathematical

Sciences
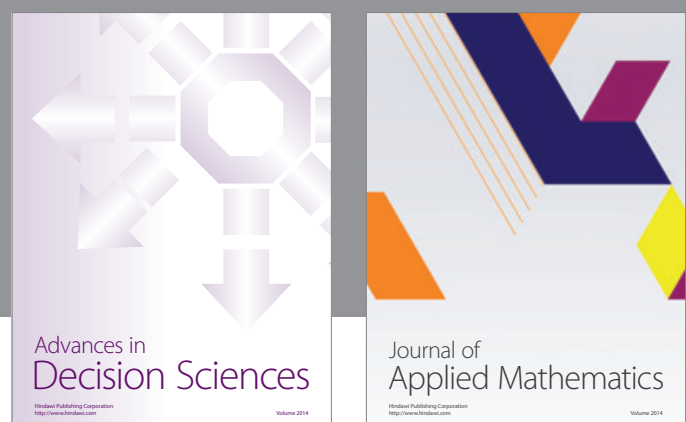

Journal of

Applied Mathematics
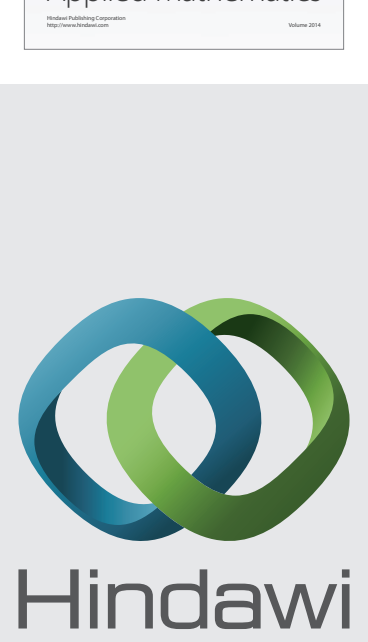

Submit your manuscripts at http://www.hindawi.com
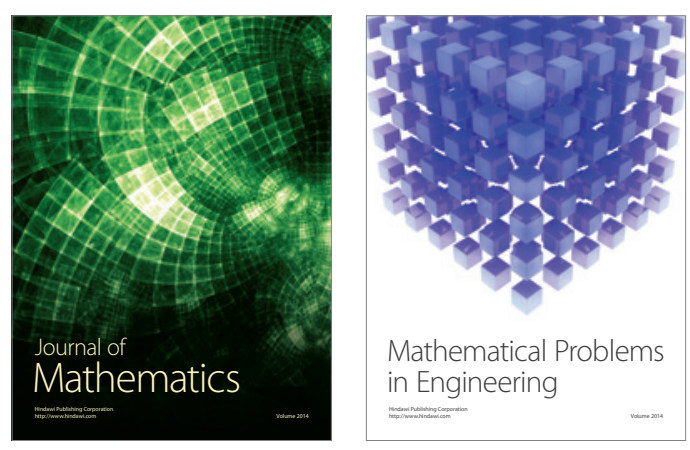

Mathematical Problems in Engineering
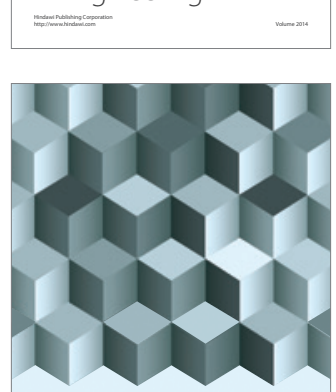

Journal of

Function Spaces
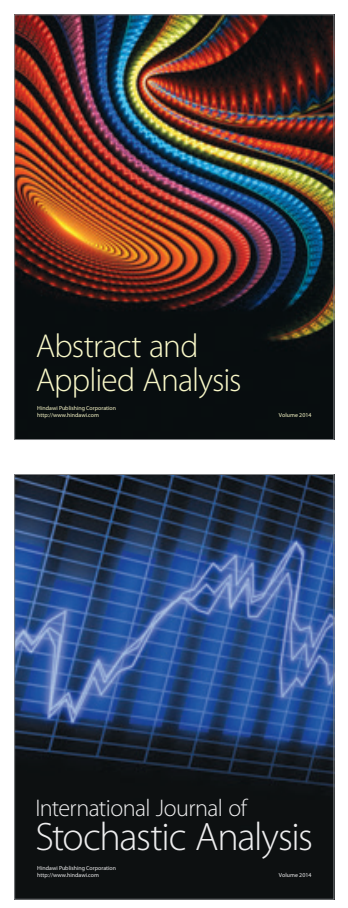

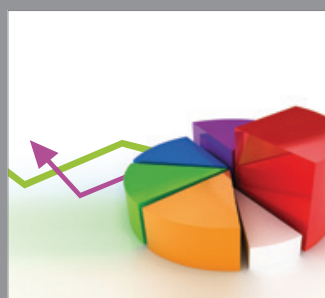

ournal of

Probability and Statistics

Promensencen
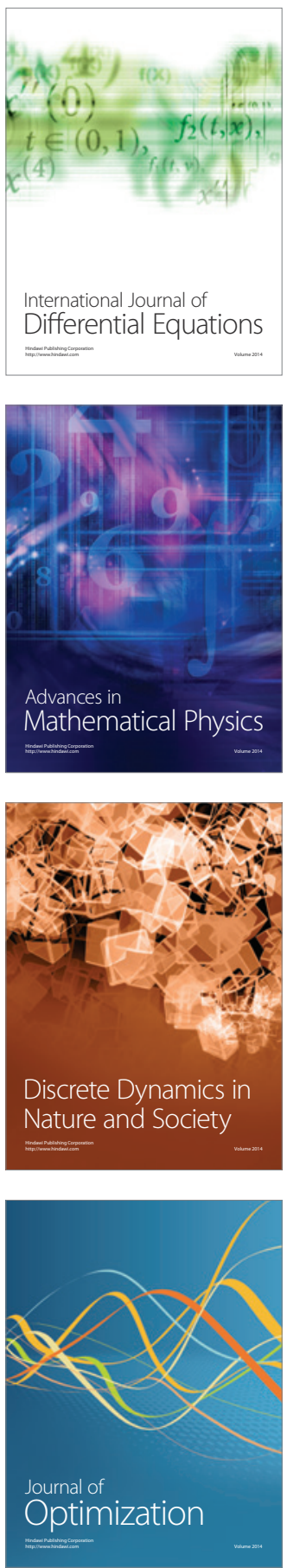